\title{
Magnetic Solitons for Non-heisenberg Anisotropic Hamiltonians in Linear Quadrupole Excitations
}

\author{
Yousef Yousefi \\ Department of Physics, Payame Noor University, Tehran, Iran
}

Email address:

Yousefi.Y@pnurazavi.ac.ir

\section{To cite this article:}

Yousef Yousefi. Magnetic Solitons for Non-heisenberg Anisotropic Hamiltonians in Linear Quadrupole Excitations. American Journal of Nano Research and Applications. Vol. 5, No. 3, 2017, pp. 37-39. doi: 10.11648/j.nano.20170503.12

Received: December 26, 2016; Accepted: January 13, 2017; Published: June 29, 2017

\begin{abstract}
We discuss system with non-isotropic non-Heisenberg Hamiltonian with nearest neighbor exchange within a mean field approximation process. We derive equations describing non-Heisenberg non-isotropic model using coherent states in real parameters and then obtain dispersion equations of spin wave of dipole and quadrupole branches for a small linear excitations from the ground state. Finally, the soliton solution for quadrupole branches for these linear equations is obtained.
\end{abstract}

Keywords: Spin System, Non-isotropic Non-heisenberg Model, Soliton Solution

\section{Introduction}

During the past decade study of nonlinear behavior of magnetic crystals has been attracted large attention, specially it accompany with the progress in some other fields such as development of theory of nonlinear differential equations, achieving new laboratory results and also potential applications in other fields of science and technology [1 - 3].

Particles with spin $S \geq 1$ are more interesting among the other nano particles $[4,5]$. This is because of existing of complexity in their behavior due to their multipole dynamic spin excitations. In such systems, the number of necessary parameters for complete description of macroscopic properties increases up to $4 S, S$ stands for magnitude of system spin.

Also it is worthwhile thst the process of achieving classical spin equations and dynamic multipoles is based on coherent states that are obtained in $S U(2 S+1)$ group $[6,7]$.

We consider unitary anisotropic Hamiltonian in the form

$$
\widehat{H}=-J \sum\left(\vec{S}_{i} \vec{S}_{i+1}+\delta \hat{S}_{i}^{z} \hat{S}_{i}^{z}\right),
$$

where $\hat{S}_{i}^{x}, \hat{S}_{i}^{y}$ and $\hat{S}_{i}^{z}$ are spin operators in the lattice $i$, and $\delta$ is the anisotropic coefficient. This is Hamiltonian of one dimensional ferromagnetic spin chain observed in compositions like $\mathrm{CSNiF}_{3}$ [8].

In this paper the goal is to obtain classical equation for stated Hamiltonian and finding the answer on question about spin wave for small linear excitations upper than the ground state. Coherent states issued nearest approximation to classical state i.e. pseudo classical, because they minimize uncertainty principles. For this reason, in the Section 2, coherent states for spin $S=1$ are developed that are the same as coherent states in $S U(3)$ group. To obtain classical Hamiltonian, we need average values of spin operator; so in the Section 3, these values and classical Hamiltonian equation are derived. In the Section 4, Hamiltonian equation computed in previous section is substituted in classical equation of motion resulted from using Feynman path integral on coherent states, and then we acquire spin wave equations and dispersion equations of dipole and quadupole branches for small linear excitation above the ground state, and finally we calculate soliton solutions of linearized equations.

\section{Coherent States in $S U(3)$ Group}

Coherent states are special quantum states, dynamic of which is very similar to behavior of their classical systems. The kind of coherent state that is used in a problem depends on the existent operators' symmetry. With considering existent symmetry in operators of Hamiltonian (1), coherent states in $S U$ (3) group is used for accurate description and considering all multipole excitations. In this group, ground state considered as $(1,0,0)^{T}$ and its single-site coherent state is written as $[9,10]$ :

$$
|\psi\rangle=D^{1}(\theta, \varphi) e^{-i \gamma \hat{S}^{z}} e^{2 i g \hat{Q}^{x y}}|0\rangle .
$$

In above equation, $D^{1}(\theta, \varphi)$ is the Wigner function for 
spin $S=1$ and two angles $\theta$ and $\varphi$ determine alignment of classical spin vector. Angle $\gamma$ determines the direction of quadruple torque around the spin vector. Parameter $g$ specifies change of length of average value of quadruple torque and also of magnitude of spin vector. Lagrangian can be obtained by use of Feynman path integral for declared coherent states as:

$$
L=\cos 2 g\left(\cos \theta \varphi_{t}+\gamma_{t}\right)-H(\theta, \varphi, \gamma),
$$

where $x_{t}=\partial / \partial t$ and $H$ is classical energy of system obtained by averaging Hamiltonian (1) on coherent states (2). Two other terms appear when acquiring Lagrangian of a spin system. The first is kinetic term that has Berry phase characteristics issued from quantum interference of Instanton paths and has important role in quantum phenomena such as spin tunneling and the second is boundary term that depends on boundary values of path [11]. Both of term have no role in classical dynamic of spin excitations and so are not considered here.

\section{Classical Hamiltonian and Equations in $S U(3)$ Group}

Average spin values in $S U(3)$ group are written as:

$$
\begin{aligned}
& S^{+}=e^{i \varphi} \cos 2 g \sin \theta, \\
& S^{-}=e^{-i \varphi} \cos 2 g \sin \theta, \\
& S^{z}=\cos 2 g \cos \theta .
\end{aligned}
$$

By averaging Hamiltonian (1) and using relations (4), the continuous limit of the obtained classical Hamiltonian is:

$$
\begin{array}{r}
H_{c l}=-J \int \frac{d x}{a_{0}}\left\{\cos ^{2} 2 g+\frac{\delta}{2}\left(\cos ^{2} \theta+\sin 2 g \cos 2 \gamma \sin ^{2} \theta\right)-\right. \\
\left.\frac{a_{0}^{2}}{2}\left(\left(\theta_{x}^{2}+\varphi_{x}^{2} \sin ^{2} \theta\right) \cos ^{2} 2 g+4 g_{x}^{2} \sin ^{2} 2 g\right)\right\} .
\end{array}
$$

To obtain the classical equation of motion, the above classical Hamiltonian is substituted in motion equations resulted from Lagrangian:

$$
\begin{gathered}
\frac{1}{\omega_{0}} \varphi_{t}=\delta \cos \theta(\sec 2 g-\cos 2 \gamma \tan 2 g) \\
+a_{0}^{2} \cos 2 g\left(\theta_{x x} \csc \theta+\varphi_{x}^{2} \cos \theta\right), \\
\frac{1}{\omega_{0}} \theta_{t}=\frac{\delta}{2} \sin 2 \theta \sin 2 \gamma \tan 2 g-a_{0}^{2} \varphi_{x x} \cos 2 g \sin \theta, \\
\frac{1}{\omega_{0}} g_{t}=-\frac{\delta}{2} \sin 2 \gamma \sin ^{2} \theta, \\
\frac{1}{\omega_{0}} \gamma_{t}=\{4 \cos 2 g-\delta(\cos 2 \gamma(\cot 4 g-\cos 2 \theta \csc 4 g)+ \\
\left.\left.\cos ^{2} \theta \sec 2 g\right)\right\}+\left\{\operatorname { c o s } 2 g \left(8 g_{x}^{2}-2 \theta_{x}^{2}+\frac{1}{2} \varphi_{x}^{2}(-3+\right.\right. \\
\left.\left.\cos 2 \theta)-\theta_{x x} \cot \theta\right)+4 g_{x x} \sin 2 g\right\} a_{0}^{2} .
\end{gathered}
$$

These equations completely describe nonlinear dynamics of Hamiltonian of problem up to quadrupole excitation. Their solutions are magnetic solitons. These equations result
Landau-Lifshitz equation if quadrupole excitations are ignored $(g=0)$. So they are more general in comparison with Landau-Lifshitz equation and have more degree of freedom. It's noteworthy to say that solution of these equations has different range of solitons.

For the case of easy axis anisotropy with $\delta<0$, to find the smallest value of Hamiltonian (1) vary it respect to all parameters. The minimum of Hamiltonian is reached at $\theta=\frac{\pi}{2}, \gamma=\frac{\pi}{2}$ and $\sin 2 g_{0}=\frac{|\delta|}{4}$, where $|\delta|<4$, this points is the ground state of the system.

For small linear excitation from ground state, classical equations of motion change to:

$$
\begin{gathered}
\frac{1}{\omega_{0}} \varphi_{t}=\delta\left(\sec g_{0}+\tan g_{0}\right) \theta+a_{0}^{2} \cos g_{0} \theta_{x x} \\
\frac{1}{\omega_{0}} \theta_{t}=-a_{0}^{2} \varphi_{x x} \cos g_{0} \\
\frac{1}{\omega_{0}} g_{t}=-\frac{\delta}{2} \gamma \\
\frac{1}{\omega_{0}} \gamma_{t}=-2\left(2 \sin g_{0}+\frac{\delta}{2 \cos g_{0}}\right) g+4 g_{x x} \sin g_{0} a_{0}^{2} .
\end{gathered}
$$

To obtain dispersion equations, functions $\theta, \varphi, \gamma$ and $g$ are considered as plane waves and their substitution in linearized equations result in dispersion equation for spin wave near the ground state:

$$
\begin{gathered}
\omega_{1}^{2}=\omega_{0}^{2} k^{2} a_{0}^{2} \operatorname{cosg}_{0}\left(\delta\left(\sec g_{0}+\tan g_{0}\right)+k^{2} a_{0}^{2} \cos g_{0}\right), \\
\omega_{2}^{2}=\omega_{0}^{2} \delta\left(\frac{\delta}{\cos g_{0}}+2 \sin g_{0}\left(k^{2} a_{0}^{2}+1\right)\right) .
\end{gathered}
$$

From the above equation, it is obvious that both dipole and quadruple branches of unitary Hamiltonian are dispersive in presence of linear excitations. If the unitary anisotropy coefficient is zero $(\delta=0)$, we have only dipole dispersion branch and there is no quadruple dispersion. In other words, quadruple dispersion branch is obtained only when there is square spin term, $\left(\hat{S}_{i}^{z} \hat{S}_{i}^{z}\right)$, in Hamiltonian.

To compute soliton solutions of equations (7), we define variable $\eta$ such as $\eta=x-v t$. In this case above equations convert to below nonlinear equations:

$$
\begin{gathered}
\left(\frac{v^{2}}{\omega_{0}^{2}}+\delta a_{0}^{2}\left(1+\sin g_{0}\right)\right) \theta_{\eta}+\left(a_{0}^{2} \cos g_{0}\right)^{2} \theta_{\eta \eta \eta}=0 \\
\frac{-2}{\delta \omega_{0}} g_{t t}=-2\left(2 \sin g_{0}+\frac{\delta}{2 \cos g_{0}}\right) g+4 a_{0}^{2} g_{x x} \sin g_{0}
\end{gathered}
$$

The first equation is third order differential equation. So change of dipole moment in Hamiltonian (1) is not of the soliton form. Solution of this equation has the following forms:

$$
\theta=C \sin \left((x-v t)\left(\frac{\left(a_{0}^{2} \cos g_{0}\right)^{2}}{\left(\frac{v^{2}}{\omega_{0}^{2}}+\delta a_{0}^{2}\left(1+\sin g_{0}\right)\right)}\right)^{1 / 2}\right)
$$

In this solution, if unitary anisotropy coefficient is limited, there is no any change in its general form, but the magnitude 
of frequency oscillation changes.

The second equation is nonlinear Klein-Gordon equation and shows change of average value of quadruple excitation that its solution is of the hylomorphic solitons form. These solitons are like of $Q$-ball solitons. The reason of this name is because they cause matter, which have the appropriate form. Also these solitons are of non-topologic kind because their boundary values in ground and infinity are the same from the topological point of view.

Let's rewrite nonlinear Klein-Gordon equation (9) as:

$$
g_{t t}=\alpha g_{x x}+\beta g,
$$

where

$$
\begin{gathered}
\alpha=-\delta \omega_{0} a_{0}^{2} \sin g_{0}, \\
\beta=\frac{\delta \omega_{0}\left(4 \sin 2 g_{0}+\delta\right)}{8 \cos g_{0}} .
\end{gathered}
$$

As we seen in relations (9), (11) and (12), if we remove the unitary anisotropy coefficient $\delta$, the equations are well defined only when $g=0$. The concept of this sentence is that the quadruple excitation in this equation existed only when there is the anisotropy term in Hamiltonian or in other words quadratic spin term in Hamiltonian.

Numerical solution of (11) is plotted in Figure 1. In this computation we consider $\alpha=10^{5}$ and $\beta=10^{10}$.

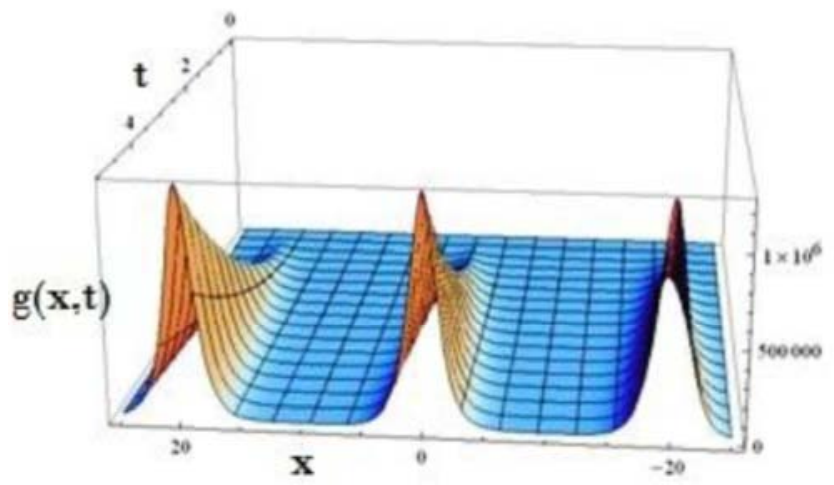

Figure 1. Numerical solution (quadruple excitation) of relation (11) is a Helomorphic soliton.

Analytical solution of above nonlinear Klein-Gordon equation is the following form:

$$
g(x, t)=C \sinh \left[(x-v t) \sqrt{\left|\frac{-\omega_{0}\left(4 \sin 2 g_{0}+\delta\right)}{\cos g_{0}\left(v^{2}+\delta \omega_{0} a_{0}^{2} \sin g_{0}\right.}\right|}\right],
$$

where $C$ is the constant.

\section{Conclusion}

In this paper, we study semi-classical theory for spin systems with spin $S=1$ that contain anisotropic exchange terms. It is shown that for anisotropic ferromagnet, value of average quadruple torque is not constant $\left(g_{t} \neq 0\right)$ and its dynamic contains rotational term around classical spin vector $\left(\gamma_{t} \neq 0\right)$ and another dynamics that relates to change of length of quadruple torque. There are no such excitations in regular magnets and their dynamics are achieved by use of average value of Heisenberg spin Hamiltonian. Also it is shown that soliton solutions are of the kind of non-topologic hilomorphic solitons for quadruple excitations.

\section{References}

[1] E. L. Nagaev, Sov. Phys. Uspekhi, vol. 25, p. 31, 1982.

[2] E. L. Nagaev, "Magnets with Non-Simple Exchange Interactions," Nauka: Moscow, 1988.

[3] V. M. Loktev and V. S. Ostrovskii, Low Temp. Phys., vol. 20, p. 775, 1994.

[4] Kh. O. Abdulloev, Kh. Kh. Muminov, Phys. Solid State, vol. 36, p. 170, 1994.

[5] Y. Yousefi and Kh. Kh. Muminov, Adv. Cond. Matter Phys., vol. 2015, p. 854625 (1-4), 2015.

[6] V. S. Ostrovskii, Sov. J. Exp. Theo. Phys., vol. 64, p. 999, 1986.

[7] Y. Yousefi and Kh. Kh. Muminov, Iranian J. Phys. Res, vol. 12, p. 179, 2012.

[8] L. Mead and N. Papanikolaou, Phys. Lett., vol. 41, p. 1137, 1978.

[9] Kh. O. Abdulloev and Kh. Kh. Muminov, Reps. Acad. Sci. Rep. Tajikistan, vol. 36, p. 6, 1993.

[10] Kh. O. Abdulloev and Kh. Kh. Muminov, Proc. Tajikistan Acad. Sci., vol. 1994-1, p. 28, 1994.

[11] Kh. Kh. Muminov and Y. Yousefi, Reps. Acad. Sci. Rep. Tajikistan, vol. 57, p. 660, 2014. 